\title{
Electron-microscopic Analysis of Kerogens in Shale Oil Rocks
}

XiaOQi Wang, Xu Jin, Hang JiaO, WeIPENG Yan, JiANMING Li, Ling SU, XIAODAN LIU AND SiwEI MENG

Research Institute of Petroleum Exploration and

Development, PetroChina, Beijing 100083, China

Shale oil is one of the most promising unconventional oil resources for comercial use in China, due to the fast development of crutial technologies including horizontal well - hydraulic fracturing and in-situ heating conversion. However, to achieve the real successful usage of shale oil, the oil in the matrix nanapores should be connected and extracted, while the majority of oil is absorbed in the kerogens, instead of free oil. Therefore, large efforts should be made to investigate the micro-structure and oil absorption of the kerogen, and also the in-situ thermal evoltion process of kerogen. In this work, we applied multi-methods of electron microscopy, including focused ion beam - scanning electron microscope (FIB-SEM), Raman-SEM, in-situ heating SEM and transmission electron microscope (TEM) to characterize the structure of kerogens in shale oil samples from Erdos, Songliao, and Jungar basins in China, and shale oil samples from USA and Mid-East.

All the shale samples with the maturity in the oil generating window, which were originally considered good source rock samples, the residual oil distribution in the kerogen was revealed by FIB-SEM controlled local charging methods after ion polishing. The results indicated that large amount of shale oil were absorbed inside the porous kerogen particles, which corresponds to the main portion of chloroform bitumen $\mathrm{A}$ in the rock. While after the polar solvent extraction (dichloromethane and methanol mixture), the charging effects totally disappeared and tiny shrinkage cracks inside the kerogen particle appeared rather than isolated visible pores, indicating that the oil evenly distributed in the kerogen particle. The TEM images also showed a homogeneous amorphous structure with low maturity, demonstrating the uniformity of adsorbed oil distribution. The Raman-SEM tests show that the laser beam will induce a heating ablation phenomenon. With the power and time duration of irradition increase, the $\mathrm{I}_{\mathrm{D}} / \mathrm{I}_{\mathrm{G}}$ ratios changes dramatically, indicating a thermal evolution of the kerogen, and the dissipation of absorbed oil. The in-situ SEM heating experiment directly show the thermal cracking process of kerogen, including pore generation at micron-scale and kerogen collapse. All these data give more information than conventional organic petrology and organic geochemistry. 